\title{
Comparing central pain processing in individuals with non-traumatic neck pain and healthy individuals: a systematic review and meta-analysis
}

Running title: Central sensitization in non-traumatic neck pain

\author{
Yanfei Xie ${ }^{\mathrm{a}}$, Deokhoon Jun ${ }^{\mathrm{a}, \mathrm{b}}$, Lucy Thomas ${ }^{\mathrm{a}}$, Brooke Coombes ${ }^{\mathrm{c}}$, Venerina Johnston ${ }^{\mathrm{a}, \mathrm{d}}$

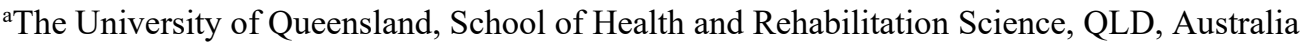 \\ ${ }^{b}$ Kyungsung University, Department of Physical Therapy, Busan, South Korea \\ ${ }^{\mathrm{c}}$ Griffith University, School of Allied Health Sciences, QLD, Australia \\ ${ }^{\mathrm{d}}$ The University of Queensland, RECOVER Injury Research Centre, QLD, Australia
}

Corresponding author: Yanfei Xie

Email: yanfei.xie@uqconnect.edu.au

Postal address: School of Health and Rehabilitation Sciences, Therapies Building 84A, level

7, The University of Queensland, St Lucia, QLD, 4072

Phone number: +61466002590

\section{Disclosures}

Authors have no conflict of interest to declare and there is no funding for this systematic review and meta-analysis. 


\begin{abstract}
This systematic review and meta-analysis examined the evidence for altered central pain processing in people with non-traumatic neck pain and the relationship between central pain processing, demographics and pain-related characteristics. Case-control studies reporting measures of altered central pain processing using quantitative sensory testing were reviewed. Standardized mean differences (SMDs) and 95\% confidence intervals (CIs) between people with non-traumatic neck pain and controls were calculated. Meta-analysis was performed using random-effects models when appropriate. Associations between SMDs with demographics and pain-related characteristics were explored on a study level using meta-regression. Twenty-six studies were eligible with 25 included for meta-analysis. Meta-analysis demonstrated mechanical hyperalgesia at remote non-painful sites in the full sample [sample size $(n)=1305$, $\mathrm{SMD}=-0.68]$ and in the subgroup with moderate/severe disability $[\mathrm{n}=165, \mathrm{SMD}=-0.86]$ (moderate-quality evidence). Meta-regression indicated that remote mechanical hyperalgesia was negatively associated with age $\left(\mathrm{R}^{2}=25.4 \%, P=0.031\right)$. Very-low- to low-quality evidence of remote cold and heat hyperalgesia and dysfunctional conditioned pain modulation were identified. This review suggests that altered central pain processing is present in people with non-traumatic neck pain and may be associated with disability levels and age.
\end{abstract}

[Word count: 179]

Perspective: This review found moderate-quality evidence of mechanical hyperalgesia at remote non-painful sites in patients with non-traumatic neck pain compared to controls, indicating altered central pain processing. However, more studies are needed to confirm findings from dynamic quantitative sensory testing.

[Word count: 39]

Keywords: nonspecific neck pain, idiopathic neck pain, central sensitization, meta-analysis, quantitative sensory testing 


\section{Introduction}

Neck pain is prevalent ${ }^{40}$ and up to $80 \%$ of people with this problem experience long-term pain and disability. ${ }^{10}$ Globally, this problem ranked the fourth greatest cause of disability out of 291 conditions studied in the Global Burden of Disease 2010 Study $^{39}$, with around 29 million disability-adjusted life-years reported in $2016 .{ }^{28}$ Neck pain is generally classified into traumatic (whiplash-associated disorders) and non-traumatic neck pain. Most people suffer from nontraumatic neck pain, which is defined as pain in the neck and/or shoulder regions (with or without referral of pain into the upper limb(s)) without trauma or specific diseases at the onset of pain development. ${ }^{34,40}$ Despite high prevalence of neck pain ${ }^{40}$, the underlying mechanisms contributing to its development and persistence remain unclear.

Altered central pain processing or central sensitization represents pathophysiological functioning of circuits and neurons within the central nervous system. ${ }^{52}$ It is thought to play an important role in many musculoskeletal pain conditions including traumatic neck pain., ${ }^{3,91}$ However, it has been suggested that different pain processes may underlie traumatic and nontraumatic neck pain. ${ }^{11}$ In people with non-traumatic neck pain, the current evidence remains unclear regarding the involvement and importance of altered central pain processing. A recent longitudinal study of 171 office workers found that dysfunctional conditioned pain modulation (CPM: thought to reflect the capacity of endogenous pain inhibitory mechanisms $)^{70}$ is an important predictor for chronic non-traumatic neck pain ${ }^{80}$. Furthermore, the dysfunctional CPM remained impaired in office workers who developed neck pain compared to those who stayed healthy after one year. ${ }^{81}$ In contrast, a previous systematic review of six studies found inconsistent results regarding the presence of altered central pain processing in non-traumatic neck pain. ${ }^{58}$ However, only a small number of studies were available when the previous review was conducted, and this review did not conduct a meta-analysis. Thus, it was not possible to confirm the existence and magnitude of altered central pain processing in people with non- 
traumatic neck pain. Considering that altered central pain processing is usually associated with poor prognosis ${ }^{44}$ and lower quality of life $^{83}$, and demands different medical and therapeutic interventions ${ }^{96}$, an up-to-date meta-analysis is warranted to advance previous work and to further clarify whether central alterations are in fact present in non-traumatic neck pain and to what magnitude.

Although a gold standard is lacking, quantitative sensory testing (QST) has been widely used as quantifiable experimental measures to evaluate indirectly the presence and nature of altered central pain processing. This includes static QST involving the measurement of pain responses to normally innocuous and noxious stimuli, including mechanical, thermal and/or electrical stimuli. ${ }^{19}$ Static QST is commonly performed at local painful and remote non-painful sites. Sensory alterations at remote non-painful sites such as allodynia (pain produced in response to innocuous stimuli) and hyperalgesia (greater sensitivity to noxious stimuli) are thought to reflect mechanisms of central pain processing. ${ }^{9,20,61}$ Localized sensory changes, however, may not be indicative of central pain processing. ${ }^{49}$ More recently, dynamic QST has been used to evaluate the complex dynamics involved in central pain processing. For example, CPM, exercise-induced analgesia paradigms and offset analgesia are thought to assess endogenous inhibitory pathways ${ }^{19,70}$, temporal and spatial summation of pain provide information about facilitatory pathways and central integration ${ }^{19,}{ }^{95}$, while nociceptive flexion reflex evaluates sensitization of spinal nociception. ${ }^{88}$

Some variabilities in findings between studies on central pain processing in nontraumatic neck pain may be associated with demographic and pain-related characteristics of the participants involved, such as age, sex, pain intensity, disability levels, and pain duration. For example, sex and age affect the size of differences between people with and without chronic pain in the efficacy of CPM. ${ }^{55}$ Among people with knee pain ${ }^{2,26}$ and unilateral lateral epicondylalgia ${ }^{15}$, those with more severe pain and disability have greater hyperalgesia 
spreading to the remote site and dysfunctional pain modulation. The effect of musculoskeletal pain duration on central pain processing is not clearly delineated in the literature. Some studies found central sensitization was only present in chronic but not (sub)acute musculoskeletal pain $^{33}$, and longer duration of pain was associated with a greater extent of central alteration. ${ }^{2}$, 33 Other studies found that signs of central alteration such as remote hyperalgesia or dysfunctional CPM can occur in the (sub)acute stage of, for example, low back pain ${ }^{48}$ and traumatic neck pain ${ }^{21}$. To further investigate and increase knowledge regarding the role of central mechanisms in non-traumatic neck pain, it is necessary to examine the potential influence of participants' characteristics on measures of central pain processing.

The primary aim of this systematic review and meta-analysis was to quantify the differences between individuals with and without non-traumatic neck pain in central pain processing as measured by QST. The secondary aim was to examine if such differences were associated with demographics and pain-related characteristics in the patient group. As such, this systematic review and meta-analysis would provide further insight into the role of central mechanisms in non-traumatic neck pain and therefore have implications for designing targeted interventions.

\section{Methods}

This systematic review has been registered in the International Prospective Register of Systematic Reviews (PROSPERO) (registration number: CRD42017081007), and has been prepared according to the Preferred Reporting Items for Systematic Reviews and MetaAnalyses (PRISMA) checklist. ${ }^{62}$

\section{Eligibility criteria}

The PICO approach was applied to establish the eligibility criteria. Eligible articles for the systematic review were original studies that examined central sensitization using static QST performed at remote sites and dynamic QST (Outcome; O) in patients with non-traumatic neck 
pain of any duration (Population; P) compared to healthy and pain-free individuals (Comparison; C). The remote site was defined as a site that is anatomically distant from the neck or shoulder regions. Any reported forms of non-traumatic neck pain were eligible for this review, including idiopathic, nonspecific, insidious, mechanical, or work-related neck pain or trapezius myalgia. Trapezius myalgia was included because its definition in the literature is similar to that of non-traumatic neck pain. ${ }^{22,65}$ Studies on a mixed population (e.g. whiplash associated disorder and non-traumatic neck pain) were also included as long as results were reported separately for at least one group with non-traumatic neck pain. Furthermore, considering that individuals with neck pain usually have concurrent pain in other body regions ${ }^{45}$, studies on a combination of neck and upper limb pain were included if neck pain was the main complaint and/or neck pain was predominant in the population (e.g. $>50 \%$ of the population). To enable an exploration of the effect of demographic and pain-related characteristics on the effect size of group differences in outcome measures of central pain processing, there was no restriction on participants' age and pain duration. There was no limitation in publication language and year.

Exclusion criteria were studies: (1) on whiplash associated disorders, experimental or post-surgical neck pain, or neck pain resulting from specific neck diseases such as cervical disc herniation, radiculopathy, and myelopathy; (2) on self-reported questionnaires of central sensitization, such as central sensitization inventory, or measures of sensory detection thresholds (defined as the point changed from no sensation to the first perceived sensation); (3) comparing cases to reference values without measuring a healthy control group; (4) abstracts for which full-text reports were not retrievable, letters, editorials, case reports, reviews, or meta-analysis.

To be included for the meta-analysis, one more criterion was applied: at least two studies provided sufficient data on the same measure of central pain processing, including mean or 
median, and standard deviation (SD), standard error, range, interquartile range or 95\% confidence interval (CI). If there were insufficient data to be included for meta-analysis, emails were sent to the authors of the respective articles to obtain further data. If no reply was received after three attempts within one month, the article was excluded from the meta-analysis. No studies were excluded for the meta-analysis based on the level of risk of bias.

\section{Information source and search strategy}

Pubmed, Web of Science, CINAHL, Embase, and PsycINFO were comprehensively searched. Search terms (MeSH and/or text word search terms) pertaining to neck pain, and terms that referred to either a state or method of testing of central pain processing were used (Table 1). Search terms were identified and developed using key articles known to the authors, and then modified and finalized after pilot searches in Pubmed to strike a balance between sensitivity and precision of searches. Reference lists of included studies were systematically searched for additional relevant studies. Additionally, a citation search was conducted using Google Scholar to identify all relevant articles that made reference to the eligible articles. The last searched date was 11 October 2019.

\section{Study selection}

Two authors (YX and DJ) independently screened title and abstracts, and then reviewed full texts independently to identify articles that met the eligibility criteria. Any disagreements were firstly discussed and resolved within the two authors (YX and DJ). If consensus could not be reached, a third author $(\mathrm{BC})$ made the final decision.

\section{Assessment of risk of bias}

Risk of bias was assessed using the Newcastle-Ottawa Scale (NOS) ${ }^{94}$ recommended by the Cochrane Collaboration for observational studies. ${ }^{73}$ As studies included for this review compared differences between cases with non-traumatic neck pain and healthy controls, the 
NOS case-control checklist was used. This checklist uses a star rating system to judge risk of bias based on selection and comparability of groups, and ascertainment of the outcome of interest. The item assessing non-response rate in the checklist was not used, because it was not applicable to all reviewed studies. Details about when the reviewed study was awarded a star for an item in the NOS were described in Supplementary 1. A maximum of eight stars can be awarded. Studies scoring $\geq 6 / 8$ were considered low risk of bias, 4-5/8 considered moderate risk of bias, and $\leq 3 / 8$ considered high risk of bias. ${ }^{26}$ Two authors ( $\mathrm{YX}$ and DJ) assessed the risk of bias in the included studies independently. Any differences in the individual NOS items were firstly discussed and resolved within the two authors (YX and DJ). If agreement could not be reached, a third author $(\mathrm{BC})$ made the final decision. The level of agreement between the two authors was determined using the Kappa coefficient: $<0.5$ indicates a low, 0.5 to 0.7 a moderate, and $>0.7$ a high level of agreement. ${ }^{13}$

\section{Data extraction}

A data extraction table agreed upon by all authors was used to extract data from included studies for evidence synthesis. Extracted data included publication details, key eligibility criteria of patients, medication use, demographics of participants, pain-related characteristics of patients (duration, pain intensity, disability levels and whether there was concurrent pain at other body regions), outcome measures of central pain processing (modalities and protocols), and main findings. To avoid repetition or double counting, for included papers that stemmed from the same study, data were extracted from the paper that provided most comprehensive information or included a larger sample size. One author (YX) extracted the data while the other author (DJ) checked the accuracy of the data.

\section{Data analysis and synthesis}

Where sufficient data were available, meta-analysis was performed using Review Manager (RevMan) (Version 5.3. Copenhagen: The Nordic Cochrane Centre, the Cocharne 
Collaboration, 2014). Standardized mean differences (SMDs) with 95\% CIs were calculated to compare patients with non-traumatic neck pain to healthy controls. For studies comparing multiple subgroups of non-traumatic neck pain (e.g. based on severity), data were combined prior to computing SMDs so that data for control participants were not double counted. An additional meta-analysis comparing the subgroup of patients with moderate/severe pain and disability to healthy controls was performed. Similarly, for studies including two control groups, data were combined to increase sample size and minimize bias. When studies measured static QST at several remote sites, data of the furthest site from the neck were used. When studies reported data on both left and right sides, data were collapsed into one value to minimize bias. For studies reporting outcomes in other formats than mean and SD (e.g. median and range), outcomes were converted to mean and SD using the computation methods recommended by Wan et $\mathrm{al}^{92}$, because this would minimise loss of precision of the metaanalysis findings and generally performs better than omitting studies ${ }^{93}$. For studies presenting data using graphs, up to three attempts were made to contact study authors via email to request the group means and SDs. If no reply was received, means and SDs were estimated from graphs in the reviewed study using the inbuilt measuring tool in Adobe Acrobat (v.9 Pro).

The SMDs were presented using forest plots. A SMD of $<0.20$ was considered negligible, $0.20-0.49$ was small, $0.50-0.79$ was moderate and $\geq 0.80$ was considered large effect in size. ${ }^{14}$ The level of significance was set at $P<0.05$. A pooled SMD was calculated using a randomeffects model if the following criteria were met: at least two studies evaluated the same outcome measure of central pain processing and heterogeneity was not substantial. Heterogeneity was considered when the Crochran's Q test was significant $(P<0.1)$, and $\mathrm{I}^{2}$ statistic $\geq 75 \%$ suggests substantial heterogeneity. ${ }^{38}$ Inverse variance weighting was used for the meta-analysis, which gave more weight to studies with a larger sample size. Publication bias was examined using a funnel plot and an Egger's test. 
Considering that some of the decisions made for the meta-analysis may affect the results, sensitivity analyses were conducted to determine the robustness of the meta-analysis. ${ }^{23}$ The influence of the following factors was examined by re-running the analysis excluding studies with: moderate or high risk of bias, no blinding of assessment, certain characteristics of populations (e.g. studies specific to adolescents or elderly, non-chronic neck pain, or nonlocalized neck pain), different definitions of the remote site (e.g. studies using the forearm as a remote site), different protocols for a same measure, and reporting medians and ranges.

For outcome measures that were not appropriate to be combined using meta-analysis, summaries of results were presented in a narrative format.

\section{Meta-regression}

If sufficient data were available ( $\geq 10$ studies providing sufficient data on the same outcome measure), random-effects meta-regression (weighted by the study sample size) was conducted using Stata (version14) to assess the effect of exploratory variables, including age, sex, pain duration, pain intensity and neck disability index, on the SMD of the outcome measures. Considering the modest number of studies available, each exploratory variable was tested in a separate univariate meta-regression. ${ }^{4}$ To increase power, when studies had subgroups of patients with non-traumatic neck pain, data from each subgroup were entered separately into the meta-regression.

\section{Assessment of the overall quality of evidence}

Quality of evidence was assessed by an approach modified from the Grading of Recommendations, Assessment, Development, and Evaluation (GRADE) criteria. ${ }^{5}$ As all reviewed studies were observational studies, the quality of evidence commenced as low quality (meaning, "further research is very likely to have an important impact on confidence in estimate of effect and is likely to change the estimate"). ${ }^{5}$ Considering study limitations (risk of bias), imprecision, inconsistency, indirectness and publication bias, quality was downgraded to very 
low quality (meaning, "very little confidence in the effect estimate") $)^{5}$ (Supplementary 2). Due to insufficient possibilities to upgrade observational studies using the original GRADE criteria $^{74}$, criteria used for upgrading were adapted from that used in a Cochrane exemplar systematic review ${ }^{35}$ according to suggestions from a previous study. ${ }^{74}$ Considering moderate or large effects, consistency, and exposure-gradient responses (Supplementary 2), quality of evidence was upgraded to moderate (meaning, "further research is likely to have an important impact on confidence in estimate of effect and may change the estimate"), or high quality (meaning, "further research is very unlikely to change our confidence in the estimate of effect").

\section{Results}

In total, 30 papers met the eligibility criteria, reporting results from 26 case-control studies (Fig. 1). Some papers stemmed from the same study and they were referenced as a single study as follows: Coppieters et $\mathrm{al}^{16-18}$, Gerdle et $\mathrm{al}^{30,76}$, and Ghafouri et $\mathrm{al}^{31,46}$ (some papers were written by different authors, but represented data of the same study population). For these papers, only data from Coppieters et $\mathrm{al}^{17}$, Gerdle et $\mathrm{al}^{76}$, and Ghafouri et al ${ }^{31}$ were extracted and included for analysis. In total, 25 studies (29 papers) were included in the metaanalysis (Fig. 1). However, for different outcomes of interest, different numbers of studies were eligible for meta-analyses (described below).

\section{Study characteristics}

Most of reviewed studies $(\mathrm{n}=24)$ recruited individuals aged between 18 to 64 years (Table 2). Of the other two studies, one only recruited adolescents $(<18 \text { years old })^{77}$ while the other only recruited the elderly ( $\geq 65$ years old).${ }^{90}$ Participants were all males in one study ${ }^{47}$, and all females in ten studies. ${ }^{17,30,31,36,43,51,65,72,82,90}$ Most studies ( $\left.\mathrm{n}=20\right)$ recruited patients with pain for at least three months (eligibility criteria) and with at least mild disability. Only two studies ${ }^{8,43}$ classified patients into groups of different levels of pain and disability. Nine 
studies investigated patients with localized neck pain ${ }^{7,8}, 11,12,36,56,72,78,81$, while ten studies investigated patients with concurrent pain in other body regions such as upper limbs $30,43,51,54$,

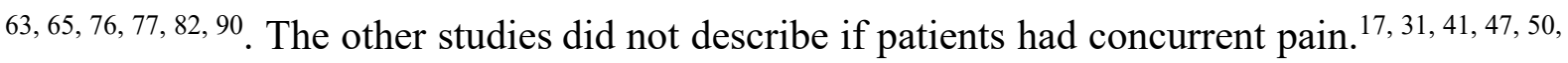
${ }^{57,84}$ Regarding the control group, most studies $(\mathrm{n}=20)$ recruited healthy individuals without any history of pain in the past year, while some studies $(n=6)$ recruited patients with occasional neck and/or other musculoskeletal pain. Most studies did not provide a clear description regarding medication use. Two studies ${ }^{7,17}$ only recruited patients reporting stable medication intake, and three studies ${ }^{54,63,72}$ excluded participants with regular or current use of medications. Participants in six studies ${ }^{30,31,51,54,87,90}$ were asked to refrain from taking medications such as analgesics and non-steroidal anti-inflammatory drugs three or seven days prior to or on the testing day.

\section{Risk of Bias of included studies}

The initial agreement rate for risk of bias assessment was 80\% (192 out of 240 items) with a Kappa coefficient of 0.64 , reflecting moderate agreement between the two raters (YX and DJ). The disagreement occurred mainly because of reading errors and divergent interpretation between two reviewers. The agreement rate reached $100 \%$ after discussion between the two raters. Eleven studies (42\%) were rated as low, $13(50 \%)$ were moderate, while two (8\%) were high risk of bias (Table 3). More than half of the studies (54\%) recruited patients with non-traumatic neck pain through convenience sampling (e.g. from a local clinic/institute that was easily accessible to researchers), resulting in potential sampling bias. Only ten studies (38\%) indicated that the investigator who assessed the outcome measures was blinded to healthy control and patient status. Most studies matched the patient and control group for age and sex, although the majority of studies $(73 \%)$ did not consider other confounders such as psychological factors.

\section{Meta-analysis and qualitative results}


Results are presented under headings according to the outcome measures of central pain processing employed. Meta-analysis was conducted for pressure pain threshold (PPT), cold pain threshold (CPT), heat pain threshold (HPT) and CPM. Regarding other outcome measures, meta-analysis was not possible due to insufficient data (e.g. lack of group means and SDs), only one study available, or heterogeneity. No reviewed studies measured allodynia, spatial summation, offset analgesia, or nociceptive flexion reflex threshold.

\section{Remote PPT}

Twenty-four studies (11 low, 11 moderate and 2 high risk of bias) tested remote PPT using a pressure algometer, which was the most common test used to assess the presence of altered central pain processing. Most studies (79\%) used the tibialis anterior as a remote site (Table 2). The quadriceps ${ }^{17,54}$, the foot ${ }^{87}$ and the extensor radialis brevis muscle ${ }^{12,36}$ were other chosen remote sites. All studies were included in the meta-analysis (693 patients versus 612 controls), except for one study ${ }^{87}$ due to a lack of SDs of remote PPT for both groups. This small study ( 8 patients versus 31 controls) found no significant group differences in PPT at the foot. ${ }^{87}$ The pooled SMD was -0.68 (95\% CI: -0.86 to -0.49 ; Fig. 2A), representing a moderate effect size of remote mechanical hyperalgesia in patients. Moderate heterogeneity was present $\left(\mathrm{I}^{2}=\right.$ $59 \%, P<0.001)$. The SMD remained moderate and consistent across sensitivity analyses

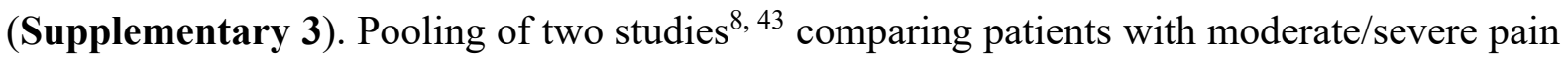
and disability $(\mathrm{n}=78)$ to healthy controls $(\mathrm{n}=87)$ demonstrated a large SMD of $-0.86(95 \% \mathrm{CI}$ : -1.22 to -0.51 ; Fig. $2 B$ ). The quality of evidence was moderate due to double upgrades for a moderate effect size and consistency and a downgrade for study limitation (Table 4).

\section{Remote CPT}

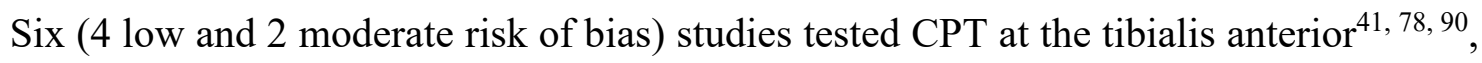
thigh $^{54}$, dorsal foot ${ }^{87}$ and dorsal hand. ${ }^{11}$ Four studies (90 patients versus 87 controls) were included in meta-analysis. Heterogeneity was low $\left(\mathrm{I}^{2}=30 \%, P=0.230\right)$. The meta-analysis 
demonstrated a SMD of 0.51 (95\% CI: 0.14 to 0.89 ; Fig. 3A), representing a moderate effect size of remote cold hyperalgesia in patients. However, the pooled SMD became insignificant in sensitivity analyses when excluding a study of moderate risk of bias ${ }^{11}$ or a study on the elderly population ${ }^{90}$ (Supplementary 3). Two studies ${ }^{54,87}$ were not included for meta-analysis because the group means, SDs or 95\% CIs of remote CPT were not available. For these two studies, one study ${ }^{87}$ found remote cold hyperalgesia in patients while the other study ${ }^{54}$ showed no group differences. Overall, the quality of evidence remained low after an upgrade for an overall moderate effect size and a downgrade for inconsistency (Table 4).

\section{Remote HPT}

Five (4 low and 1 moderate risk of bias) studies assessed HPT at the tibialis anterior ${ }^{41,78,}$ ${ }^{90}$, thigh ${ }^{54}$ and dorsal foot. ${ }^{87}$ All studies (80 patients versus 97 controls) were included in the meta-analysis. The SMD was -0.37 (95\% CI: -0.70 to -0.05 ; Fig. 3B), representing a small effect size of remote heat hyperalgesia in patients. Heterogeneity was low $\left(\mathrm{I}^{2}=5 \%, P=0.380\right)$. Sensitivity analyses showed that the pooled SMD became insignificant after excluding a study on the elderly population ${ }^{90}$ (Supplementary 3). As a result, the quality of evidence was downgraded to very low quality (Table 4).

\section{Other static QST measures at remote sites}

One low risk of bias study tested mechanical pain threshold at the dorsal foot using a set of pinprick stimulators with fixed intensities ranging from 8 to $512 \mathrm{mN}$, reporting no differences between patients $(n=8)$ and controls $(n=31) .{ }^{87}$ One moderate risk of bias study assessed pressure pain tolerance (the point at which the participant felt the pain as intolerable) remotely at the lower leg using a computer-controlled cuff-algometer, demonstrating lower pressure pain tolerance in patients than healthy controls $(\mathrm{SMD}=-0.78,95 \% \mathrm{CI}:-1.43$ to 0.12). ${ }^{36}$ Both measurements of mechanical pain threshold and pressure pain tolerance had verylow quality of evidence due to imprecision (Table 4). 
Four moderate risk of bias studies ${ }^{17,36,54,81}$ examined CPM. The conditioning stimulus was induced by ischaemic pain at the calf $f^{36}$ or $\operatorname{arm}^{54}$, or cold water immersion of the hand. ${ }^{17,81}$ The outcome measures analysed were absolute and/or percentage changes of PPT at the painful/dominant trapezius and/or lower $\operatorname{leg}^{17,36,54,81}$, thermal pain threshold ${ }^{54}$ or suprathreshold heat pain at the right thigh ${ }^{54}$ before compared to during conditioning stimulus. Three studies (71 patients versus 58 controls) were included in the meta-analysis, demonstrating a small effect size (SMD: $-0.36,95 \% \mathrm{CI}$ : -0.72 to -0.01 ) of lower efficacy of CPM in patients compared to healthy controls (Fig. 3C). No heterogeneity between three studies $\left(\mathrm{I}^{2}=0 \%, P=\right.$ 0.470) was observed. In the sensitivity analysis, the SMD became insignificant after excluding the study ${ }^{37}$ using ischaemic pain as the conditioning stimulus (Supplementary 3). One study ${ }^{54}$ was not included in the meta-analysis due to insufficient data. The sample size of this study was small (10 patients versus 10 healthy controls) and no group differences were found. ${ }^{54}$ The overall quality of evidence for CPM was downgraded to very low due to study limitation and inconsistency (Table 4).

\section{Exercise-induced analgesia}

Two studies ${ }^{12,} 47$ with moderate risk of bias applied exercise-induced analgesia paradigms. Meta-analysis was not possible because of heterogeneity of the methodology. Christensen et $\mathrm{a}^{12}$ found that compared to healthy controls, patients with localized chronic non-traumatic neck pain had less efficacy of exercise-induced analgesia, as they showed significantly increased pain sensitivity and intensity after three sets of slow and fast arm movements. In contrast, Kemppainen et $\mathrm{al}^{47}$ showed that 20 to 32 minutes of lower limb aerobic exercise induced statistically longer time to pain onset during the cold pressor test and greater reduction in pain intensity and unpleasantness in response to supra-threshold cold stimulation in patients than health controls. This demonstrated that patients with a history of acute neck 
pain attacks had greater efficacy of exercise-induced analgesia. However, the patient group in the study from Kemppainen et $\mathrm{al}^{47}$ did not have any pain at the time of testing, and no detailed information was provided about how significant a neck problem it was. Because of the conflicting results reported and large variation of the methodology, evidence was inconclusive regarding the case-control difference in the exercise-induced analgesia efficacy. Such evidence was evaluated to be very low quality (Table 4).

\section{Temporal summation of pain}

One moderate ${ }^{36}$ and one low risk ${ }^{87}$ of bias studies examined the wind-up ratio to either repetitive pinprick or cuff pressure stimuli as a measure of temporal summation. Stimuli were applied to the neck/upper thoracic area ${ }^{87}$, the corresponding dermatome ${ }^{87}$, the foot $^{87}$ or the lower leg. ${ }^{36}$ A meta-analysis was not possible due to insufficient data (lack of SDs for groups)

provided by one study. ${ }^{87}$ Both studies concluded no significant group differences in temporal summation. Such evidence was evaluated to be very low quality (Table 4).

\section{Assessment of publication bias}

Publication bias was assessed for each meta-analysis. Visual analyses of funnel plots showed reasonably symmetrical graphs (Fig. 4) and Egger's tests confirmed that no publication bias was observed for remote PPT $(P=0.507)$, CPT $(P=0.990)$, and HPT $(P=0.970)$, and $\operatorname{CPM}(P=0.300)$.

\section{Meta-regression}

Meta-regression was possible for analysing associations of remote PPT with the patient's age, percentages of female patients, neck disability index and pain duration. Meta-regression was not possible for analysing the association between remote PPT and pain intensities, as pain intensities of different periods were recorded across studies (some reported average or maximum pain levels last week while some reported current pain levels). Age was negatively 
associated with SMDs of remote PPT [number of observations $(\mathrm{n})=28, P=0.031$, adjusted $\mathrm{R}^{2}$ $=25.4 \%$. The study with elderly patients ${ }^{90}$ showed the smallest effect size, while the study with adolescents ${ }^{77}$ displayed the largest effect size. The effect size of remote PPT was not correlated with other variables, including percentages of female patients $(\mathrm{n}=28, P=0.302)$, neck disability index $(\mathrm{n}=20, P=0.317)$ or pain duration $(\mathrm{n}=21, P=0.350)$.

\section{Discussion}

\section{Group differences in outcome measures of central pain processing}

This is the first review to quantify the differences in measures of central pain processing using meta-analytic techniques, involving 693 patients with non-traumatic neck pain and 612 healthy controls. We identified moderate-quality evidence of remote mechanical hyperalgesia in patients with non-traumatic neck pain, demonstrated by significantly reduced PPT at remote sites that are not directly affected by the neck pain. Such a finding was statistically robust and pooled SMDs were moderate across all sensitivity analyses, suggesting the group difference could be clinically meaningful, although there is no consensus about the magnitude of differences in PPT required to be clinically meaningful. ${ }^{85}$ Additionally, remote thermal (cold and heat) hyperalgesia and dysfunctional CPM were also demonstrated in patients with nontraumatic neck pain. These findings are indicative of central alterations in non-traumatic neck pain.

Remote hyperalgesia is usually accepted as an indicator of central sensitization. ${ }^{9,}$ 20, 61 Repetitive or prolonged peripheral sensitization, a well-documented sensory change of nontraumatic neck pain ${ }^{8,41,43,50,51,77,82,90}$, can initiate a gradual increase of nociceptive input from for example, tight neck and shoulder muscles or active myofascial trigger points ${ }^{1,25}$ to the spinal cord, eliciting sensitization in the central nervous system. ${ }^{6}$ When neurons in the spinal cord or further upstream are subject to central sensitization, they can exhibit an expansion or

enlargement of their receptive fields, enabling the occurrence of remote hyperalgesia. ${ }^{52}$ 
Therefore, hyperalgesia spreading to the remote non-painful site is likely mediated by alteration of central pain processing rather than a simple extension of peripheral hyperalgesia or inflammation. $^{52}$

Dysfunctional CPM, which is thought to indicate descending pain inhibition through activating endogenous analgesia pathways ${ }^{19,70}$ was evident in non-traumatic neck pain. In contrast, enhanced temporal summation, which may reflect increased excitability of spinal cord neurons ${ }^{19,95}$ was not supported. Different sensory stimuli (e.g. pressure and thermal pain thresholds) and different dynamic protocols may provide unique information on distinct pathways of pain perception ${ }^{64}$, or involvement of different spinal, brainstem or cortical levels .${ }^{19,89}$ However, this possibility needs to be further confirmed in future studies, as this review did not include any brain measures such as measures of central activity using functional MRI.

Factors such as stress ${ }^{42}$, depressive symptoms ${ }^{27}$, pain catastrophizing ${ }^{60}$, alcohol drinks ${ }^{48}$ and sleep disturbance ${ }^{48}$ could also contribute to altered central pain processing, although were not considered in many of the reviewed studies. Hence, the contribution of these cognitive, affective, social and lifestyle factors to central pain processing in non-traumatic neck pain remains to be determined.

An alternative explanation for the discrepancy in results may also be due to methodological issues including but not limited to sample size, quality of studies and different protocols used. Perhaps because extensive QST protocols are difficult to apply, and the algometer is an easy-to-use instrument for measuring PPT, many reviewed studies only used a single measure of PPT, while a small number of studies used dynamic QST with different protocols. Furthermore, some other QST measures such as allodynia and nociceptive flexion reflex measures were not measured in the reviewed studies, although they might provide some more meaningful information about central pain processing. Future investigations are 
recommended to use more than one type of QST measure to further clarify the evidence of specific central pain mechanisms in patients with non-traumatic neck pain. Moreover, to enable comparison between studies and reach a firm conclusion, future studies on dynamic QST such as CPM should consistently follow the most recent recommended protocols..$^{97}$

\section{Effect of demographics and pain-related characteristics}

Effects of the patient's age, the percentage of female patients, neck pain duration, and disability levels on the effect size of remote PPT were explored using meta-regression. Age was the only factor that was significantly associated with the effect size of remote PPT, with the largest effect size evident in the study involving adolescents and the smallest effect size in the study on elderly populations. Previous studies reported decreased pain threshold and reduced efficacy of endogenous analgesic systems with age due to the age-related alterations in the structure and function of both peripheral and central nociceptive pathways. ${ }^{32,37,53,71}$ These age-related sensory alterations may make it difficult to find differences in the painrelated mechanical hyperalgesia in the patient group with older age compared to healthy controls. While some reviewed studies did not control for the effect of age, future studies on central pain processing should carefully match the age of the population or consider age as a confounding factor and make statistical adjustment accordingly.

Some studies ${ }^{8,41,43,50}$ reviewed in this systematic review have suggested that remote mechanical hyperalgesia is more likely present in women, or those with chronic non-traumatic neck pain and severe neck disability. In our meta-regression, these relationships were not significant. As meta-regression was performed using study level information rather than individual patient data, the lack of variability of these exploratory variables (e.g. the majority of studies included female patients with mild disability levels and chronic pain) may have resulted in insufficient power to produce significant results. However, a larger effect size 
(SMD: -0.86) was observed when the subgroup of patients with moderate/severe pain and disability were considered, suggesting that the degree of remote mechanical hyperalgesia (and thus central sensitization) might be related to disability levels. The effect size of this subgroup is comparable to that reported in other types of musculoskeletal disorders that are believed to present with altered central pain processing including whiplash associated disorders (SMD: $0.78,95 \%$ CI: -1.31 to -0.25$)^{17}$, painful osteoarthritis (SMD: $-0.88,95 \%$ CI: -1.11 to -0.65$)^{86}$, and lateral epicondylalgia (SMD: $-0.81,95 \% \mathrm{CI}:-1.42$ to -0.20$){ }^{24}$

\section{Implications for research and practice}

This review highlights several aspects that could be addressed in future research. First, study quality could be improved by experimental designs such as blinding assessors from patient's status. Furthermore, many studies did not provide a clear description of medication use and the extent of concurrent pain although evidence suggests that single site musculoskeletal pain is rare ${ }^{45}$. As medication use and the extent of concurrent pain may affect central pain processing, future studies may consider their relationships with various QST measures in patients with non-traumatic neck pain.

Although a clear picture was not found regarding which specific central pain pathways were dysfunctional, the current study highlights that non-traumatic neck pain may not be exclusively a peripherally driven disorder. Clinicians should be aware that altered central pain processing is likely present in particularly patients with moderate and severe neck pain and disability. Signs of altered central pain processing such as remote hyperalgesia can predict worse musculoskeletal outcomes ${ }^{29}$ and contribute to poor response to usual treatment ${ }^{44,75}$. As such, recognizing signs of altered central pain processing might lead to alternative treatments,

such as desensitizing the central nervous system. In the clinical setting, measurement of PPT has advantages of ease of implementation, and might provide useful information regarding the presence of altered central pain processing when combined with history taking ${ }^{66}$ and other 
clinical sensory tests. ${ }^{98}$ For patients with non-traumatic neck pain who present with signs of altered central pain processing, clinicians may consider shifting from treatments targeting local structures to those that target the brain and focus on different features related to central pain processing. Individually tailored multimodal or multidisciplinary therapy, for example, repeated exposure to clinically-relevant pain $^{79}$, or cognition-targeted time-contingent exercise $^{59}$ combined with psychological treatment $^{67}$, pain science education $^{59}$, sleep management ${ }^{68}$, and/or dietary modifications ${ }^{69}$ might be promising interventions in decreasing pain sensitivity and increasing pain modulatory capacity. However, further studies are needed to investigate the effectiveness of these treatments in normalizing central pain processing in patients with non-traumatic neck pain.

\section{Limitations of this review}

Some caveats are identified. First, although extensive key terms and synonyms related to neck pain and central pain processing were used, they may not be exhaustive and therefore some relevant studies may have been missed. Second, one or two studies could not be included for the meta-analysis of PPT, CPT and CPM due to insufficient data available. However, these studies had a small sample size. Therefore, they are unlikely to change the conclusions. Furthermore, there was only moderate initial inter-rater agreement on the risk of bias assessment. This may not truly reflect the risk of bias of the reviewed studies, which in turn may alter the quality of evidence found in this review. Lastly, results of the meta-regression might be limited by the small number of available studies on patients with non-traumatic neck pain with certain characteristics such as patients with acute pain.

\section{Conclusions}

This meta-analysis demonstrated that compared to healthy controls, patients with nontraumatic neck pain had a moderate effect size of remote mechanical hyperalgesia (moderatequality evidence). Such hyperalgesia was more pronounced in younger patients and in patients 
with moderate or severe symptoms. Additionally, patients with non-traumatic neck pain showed small to moderate effect sizes of remote cold and heat hyperalgesia and dysfunctional CPM, although these conclusions were based on very low to low quality of evidence. While involvement of altered central pain processing in non-traumatic neck pain is inferred from findings of this meta-analysis, additional confirmatory research is needed to examine which specific pathways within the central nervous system are involved. 


\section{References}

1. Andersen LL, Hansen K, Mortensen OS, Zebis MK. Prevalence and anatomical location of muscle tenderness in adults with nonspecific neck/shoulder pain. BMC Musculoskelet Disord 12:169, 2011. https://doi.org/10.1186/1471-2474-12-169

2. Arendt-Nielsen L, Egsgaard LL, Petersen KK, Eskehave TN, Graven-Nielsen T, Hoeck HC, Simonsen O. A mechanism-based pain sensitivity index to characterize knee osteoarthritis patients with different disease stages and pain levels. Eur J Pain 19:1406-17, 2015. https://doi.org/10.1002/ejp.651

3. Arendt-Nielsen L, Morlion B, Perrot S, Dahan A, Dickenson A, Kress HG, Wells C, Bouhassira D, Mohr Drewes A. Assessment and manifestation of central sensitisation across different chronic pain conditions. Eur J Pain 22:216-41, 2018. https://doi.org/10.1002/ejp.1140 4. Baker WL, White CM, Cappelleri JC, Kluger J, Coleman CI. Understanding heterogeneity in meta-analysis: the role of meta-regression. Int J Clin Pract 63:1426-34, 2009. https://doi.org/10.1111/j.1742-1241.2009.02168.x

5. Balshem H, Helfand M, Schunemann HJ, Oxman AD, Kunz R, Brozek J, Vist GE, FalckYtter Y, Meerpohl J, Norris S, Guyatt GH. GRADE guidelines: 3. Rating the quality of evidence. J Clin Epidemiol 64:401-6, 2011. https://doi.org/10.1016/j.jclinepi.2010.07.015

6. Baron R, Hans G, Dickenson AH. Peripheral input and its importance for central sensitization. Ann Neurol 74:630-6, 2013. https://doi.org/10.1002/ana.24017

7. Beinert K, Englert V, Taube W. After-effects of neck muscle vibration on sensorimotor function and pain in neck pain patients and healthy controls - a case-control study. Disabil Rehabil:1906-13, 2018. https://doi.org/10.1080/09638288.2018.1451925

8. Beltran-Alacreu H, López-de-Uralde-Villanueva I, Calvo-Lobo C, Fernández-Carnero J, Touche RL. Clinical features of patients with chronic non-specific neck pain per disability level: A novel observational study. Rev Assoc Med Bras (1992) 64:700-9, 2018. 
9. Burstein R, Jakubowski M, Garcia-Nicas E, Kainz V, Bajwa Z, Hargreaves R, Becerra L, Borsook D. Thalamic sensitization transforms localized pain into widespread allodynia. Ann Neurol 68:81-91, 2010. https://doi.org/10.1002/ana.21994

10. Carroll LJ, Hogg-Johnson S, van der Velde G, Haldeman S, Holm LW, Carragee EJ, Hurwitz EL, Cote P, Nordin M, Peloso PM, Guzman J, Cassidy JD. Course and prognostic factors for neck pain in the general population: results of the Bone and Joint Decade 2000-2010 Task Force on Neck Pain and Its Associated Disorders. J Manipulative Physiol Ther 32:S8796, 2009. https://doi.org/10.1016/j.jmpt.2008.11.013

11. Chien A, Sterling M. Sensory hypoaesthesia is a feature of chronic whiplash but not chronic idiopathic neck pain. Man Ther 15:48-53, 2010. https://doi.org/10.1016/j.math.2009.05.012 12. Christensen SW, Hirata RP, Graven-Nielsen T. Altered pain sensitivity and axioscapular muscle activity in neck pain patients compared with healthy controls. Eur J Pain 21:1763-71, 2017. https://doi.org/10.1002/ejp.1088

13. Cohen J. A coefficient of agreement for nominal scales. Educ Psychol Meas 20:37-46, 1960. https://doi.org/10.1177/001316446002000104

14. Cohen J. A power primer. Psychol Bull 112:155-9, 1992.

15. Coombes BK, Bisset L, Vicenzino B. Thermal hyperalgesia distinguishes those with severe pain and disability in unilateral lateral epicondylalgia. Clin J Pain 28:595-601, 2012. https://doi.org/10.1097/AJP.0b013e31823dd333

16. Coppieters I, De Pauw R, Caeyenberghs K, Danneels L, Kregel J, Pattyn A, Meeus M, Cagnie B. Decreased regional grey matter volume in women with chronic whiplash-associated disorders: relationships with cognitive deficits and disturbed pain processing. Pain Physician 20:E1025-E51, 2017.

17. Coppieters I, De Pauw R, Kregel J, Malfliet A, Goubert D, Lenoir D, Cagnie B, Meeus M. Differences between women with traumatic and idiopathic chronic neck pain and women 
without neck pain: interrelationships among disability, cognitive deficits, and central sensitization. Phys Ther 97:338-53, 2017. https://doi.org/10.2522/ptj.20160259

18. Coppieters I, De Pauw R, Caeyenberghs K, Lenoir D, DeBlaere K, Genbrugge E, Meeus M, Cagnie B. Differences in white matter structure and cortical thickness between patients with traumatic and idiopathic chronic neck pain: Associations with cognition and pain modulation? Hum Brain Mapp 39:1721-42, 2018. https://doi.org/10.1002/hbm.23947

19. Cruz-Almeida Y, Fillingim RB. Can quantitative sensory testing move us closer to mechanism-based pain management? Pain Med 15:61-72, 2014. https://doi.org/10.1111/pme.12230

20. Curatolo M, Arendt-Nielsen L, Petersen-Felix S. Central hypersensitivity in chronic pain: mechanisms and clinical implications. Phys Med Rehabil Clin N Am 17:287-302, 2006. https://doi.org/10.1016/j.pmr.2005.12.010

21. Daenen L, Nijs J, Cras P, Wouters K, Roussel N. Changes in pain modulation occur soon after whiplash trauma but are not related to altered perception of distorted visual feedback. Pain Pract 14:588-98, 2014. https://doi.org/10.1111/papr.12113

22. De Meulemeester K, Calders P, De Pauw R, Grymonpon I, Govaerts A, Cagnie B. Morphological and physiological differences in the upper trapezius muscle in patients with work-related trapezius myalgia compared to healthy controls: A systematic review. Musculoskelet Sci Pract 29:43-51, 2017. https://doi.org/10.1016/j.msksp.2017.02.007

23. Deeks JJ, Higgins JPT, Altman DG, Group obotCSM. Chapter 9: analysing data and undertaking meta-analyses. In: Higgins JPT, Churchill R, Chandler J, Cumpston MS, editor. Cochrane handbook for systematic reviews of interventions version 5.2.0

The Cochrane Collaboration2017 [updated June 2017. Available from: www.training.cochrane.org/handbook. 
24. Fernández-Carnero J, Fernández-de-Las-Peñas C, de la Llave-Rincón AI, Ge HY, ArendtNielsen L. Widespread mechanical pain hypersensitivity as sign of central sensitization in unilateral epicondylalgia: a blinded, controlled study. Clin J Pain 25:555-61, 2009. https://doi.org/10.1097/AJP.0b013e3181a68a040

25. Fernández-de-las-Peñas C, Alonso-Blanco C, Miangolarra JC. Myofascial trigger points in subjects presenting with mechanical neck pain: a blinded, controlled study. Man Ther 12:2933, 2007. https://doi.org/10.1016/j.math.2006.02.002

26. Fingleton C, Smart K, Moloney N, Fullen BM, Doody C. Pain sensitization in people with knee osteoarthritis: a systematic review and meta-analysis. Osteoarthritis Cartilage 23:104356, 2015. https://doi.org/10.1016/j.joca.2015.02.163

27. Garrigos-Pedron M, La Touche R, Navarro-Desentre P, Gracia-Naya M, Segura-Orti E. Widespread mechanical pain hypersensitivity in patients with chronic migraine and temporomandibular disorders: relationship and correlation between psychological and sensorimotor variables. Acta Odontol Scand 77:224-31, 2019. https://doi.org/10.1080/00016357.2018.1538533

28. GBD, 2016, DALYs, and, HALE, Collaborators. Global, regional, and national disabilityadjusted life-years (DALYs) for 333 diseases and injuries and healthy life expectancy (HALE) for 195 countries and territories, 1990-2016: a systematic analysis for the Global Burden of Disease Study 2016. Lancet 390:1260-344, 2017. https://doi.org/10.1016/s0140$\underline{6736(17) 32130-\mathrm{x}}$

29. Georgopoulos V, Akin-Akinyosoye K, Zhang W, McWilliams DF, Hendrick P, Walsh DA. Quantitative sensory testing and predicting outcomes for musculoskeletal pain, disability, and negative affect: a systematic review and meta-analysis. Pain 160:1920-32, 2019. https://doi.org/10.1097/j.pain.0000000000001590 
30. Gerdle B, Hilgenfeldt U, Larsson B, Kristiansen J, Søgaard K, Rosendal L. Bradykinin and kallidin levels in the trapezius muscle in patients with work-related trapezius myalgia, in patients with whiplash associated pain, and in healthy controls - A microdialysis study of women. Pain 139:578-87, 2008. https://doi.org/10.1016/j.pain.2008.06.012
31. Ghafouri N, Ghafouri
B, Larsson
B, Stensson N, Fowler
CJ, Gerdle
B. Palmitoylethanolamide and stearoylethanolamide levels in the interstitium of the trapezius muscle of women with chronic widespread pain and chronic neck-shoulder pain correlate with pain intensity and sensitivity. Pain 154:1649-58, 2013. https://doi.org/10.1016/j.pain.2013.05.002

32. Gibson SJ, Farrell M. A review of age differences in the neurophysiology of nociception and the perceptual experience of pain. Clin J Pain 20:227-39, 2004.

33. Gunnarsson HEM, Grahn B, Agerstrom J. Increased deep pain sensitivity in persistent musculoskeletal pain but not in other musculoskeletal pain states. Scand J Pain 13:1-5, 2016. https://doi.org/10.1016/j.sjpain.2016.05.032

34. Guzman J, Hurwitz EL, Carroll LJ, Haldeman S, Côté P, Carragee EJ, Peloso PM, van der Velde G, Holm LW, Hogg-Johnson S, Nordin M, Cassidy JD. A new conceptual model of neck pain: linking onset, course, and care: the Bone and Joint Decade 2000-2010 Task Force on Neck Pain and Its Associated Disorders. Spine (Phila Pa 1976) 33:S14-23, 2008. https://doi.org/10.1097/BRS.0b013e3181643efb

35. Hayden JA, Tougas ME, Riley R, Iles R, Pincus T. Individual recovery expectations and prognosis of outcomes in non - specific low back pain: prognostic factor exemplar review. Cochrane Database Syst Rev, 2014. https://doi.org/10.1002/14651858.CD011284

36. Heredia-Rizo AM, Petersen KK, Madeleine P, Arendt-Nielsen L. Clinical outcomes and central pain mechanisms are improved after upper trapezius eccentric training in female 
computer users with chronic neck/shoulder pain. Clin J Pain 35:65-76, 2019. https://doi.org/10.1097/ajp.0000000000000656

37. Hermans L, Van Oosterwijck J, Goubert D, Goudman L, Crombez G, Calders P, Meeus M. Inventory of personal factors influencing conditioned pain modulation in healthy people: a systematic literature review. Pain Pract 16:758-69, 2016. https://doi.org/10.1111/papr.12305

38. Higgins JPT, Thompson SG, Deeks JJ, Altman DG. Measuring inconsistency in metaanalyses. Bmj 327:557-60, 2003. https://doi.org/10.1136/bmj.327.7414.557

39. Hoy D, March L, Woolf A, Blyth F, Brooks P, Smith E, Vos T, Barendregt J, Blore J, Murray C, Burstein R, Buchbinder R. The global burden of neck pain: estimates from the global burden of disease 2010 study. Ann Rheum Dis 73:1309-15, 2014. https://doi.org/10.1136/annrheumdis-2013-204431

40. Hoy DG, Protani M, De R, Buchbinder R. The epidemiology of neck pain. Best Pract Res Clin Rheumatol 24:783-92, 2010. https://doi.org/10.1016/j.berh.2011.01.019

41. Javanshir K, Ortega-Santiago R, Mohseni-Bandpei MA, Miangolarra-Page JC, Fernandezde-Las-Penas C. Exploration of somatosensory impairments in subjects with mechanical idiopathic neck pain: a preliminary study. J Manipulative Physiol Ther 33:493-9, 2010. https://doi.org/10.1016/j.jmpt.2010.08.022

42. Jennings EM, Okine BN, Roche M, Finn DP. Stress-induced hyperalgesia. Prog Neurobiol 121:1-18, 2014. https://doi.org/https://doi.org/10.1016/j.pneurobio.2014.06.003

43. Johnston V, Jimmieson NL, Jull G, Souvlis T. Quantitative sensory measures distinguish office workers with varying levels of neck pain and disability. Pain 137:257-65, 2008. https://doi.org/10.1016/i.pain.2007.08.037

44. Jull G, Sterling M, Kenardy J, Beller E. Does the presence of sensory hypersensitivity influence outcomes of physical rehabilitation for chronic whiplash?--A preliminary RCT. Pain 129:28-34, 2007. https://doi.org/10.1016/j.pain.2006.09.030 
45. Kamaleri Y, Natvig B, Ihlebaek CM, Bruusgaard D. Localized or widespread musculoskeletal pain: does it matter? Pain 138:41-6, 2008. https://doi.org/10.1016/j.pain.2007.11.002

46. Karlsson L, Gerdle B, Ghafouri B, Backryd E, Olausson P, Ghafouri N, Larsson B. Intramuscular pain modulatory substances before and after exercise in women with chronic neck pain. Eur J Pain 19:1075-85, 2015. https://doi.org/10.1002/ejp.630

47. Kemppainen P, Hamalainen O, Kononen M. Different effects of physical exercise on cold pain sensitivity in fighter pilots with and without the history of acute in-flight neck pain attacks. Med Sci Sports Exerc 30:577-82, 1998.

48. Klyne DM, Moseley GL, Sterling M, Barbe MF, Hodges PW. Individual variation in pain sensitivity and conditioned pain modulation in acute low back pain: effect of stimulus type, sleep, and psychological and lifestyle factors. J Pain 19:942.e1-.e18, 2018. https://doi.org/https://doi.org/10.1016/j.jpain.2018.02.017

49. Koelbaek Johansen M, Graven-Nielsen T, Schou Olesen A, Arendt-Nielsen L. Generalised muscular hyperalgesia in chronic whiplash syndrome. Pain 83:229-34, 1999. https://doi.org/10.1016/s0304-3959(99)00106-2

50. La Touche R, Fernández-de-Las-Peñas C, Fernández-Carnero J, Díaz-Parreño S, ParisAlemany A, Arendt-Nielsen L. Bilateral mechanical-pain sensitivity over the trigeminal region in patients with chronic mechanical neck pain. J Pain 11:256-63, 2010. https://doi.org/10.1016/j.jpain.2009.07.003

51. Larsson B, Rosendal L, Kristiansen J, Sjøgaard G, Søgaard K, Ghafouri B, Abdiu A, Kjaer M, Gerdle B. Responses of algesic and metabolic substances to $8 \mathrm{~h}$ of repetitive manual work in myalgic human trapezius muscle. Pain 140:479-90, 2008.

52. Latremoliere A, Woolf CJ. Central sensitization: a generator of pain hypersensitivity by central neural plasticity. J Pain 10:895-926, 2009. https://doi.org/10.1016/j.jpain.2009.06.012 
53. Lautenbacher S, Kunz M, Strate P, Nielsen J, Arendt-Nielsen L. Age effects on pain thresholds, temporal summation and spatial summation of heat and pressure pain. Pain 115:410-8, 2005. https://doi.org/10.1016/j.pain.2005.03.025

54. Leffler AS, Hansson P, Kosek E. Somatosensory perception in a remote pain-free area and function of diffuse noxious inhibitory controls (DNIC) in patients suffering from long-term trapezius myalgia. Eur J Pain 6:149-59, 2002. https://doi.org/10.1053/eujp.2001.0312

55. Lewis GN, Rice DA, McNair PJ. Conditioned pain modulation in populations with chronic pain: a systematic review and meta-analysis. J Pain 13:936-44, 2012. https://doi.org/10.1016/j.jpain.2012.07.005

56. Linari-Melfi M, Cantarero-Villanueva I, Fernandez-Lao C, Fernandez-de-Las-Penas C, Guisado-Barrilao R, Arroyo-Morales M. Analysis of deep tissue hypersensitivity to pressure pain in professional pianists with insidious mechanical neck pain. BMC Musculoskelet Disord 12:268, 2011. https://doi.org/10.1186/1471-2474-12-268

57. López-de-Uralde-Villanueva I, Beltran-Alacreu H, Fernández-Carnero J, Kindelan-Calvo P, La Touche R. Widespread pressure pain hyperalgesia in chronic nonspecific neck pain with neuropathic features: a descriptive cross-sectional study. Pain Physician 19:77-88, 2016.

58. Malfliet A, Kregel J, Cagnie B, Kuipers M, Dolphens M, Roussel N, Meeus M, Danneels L, Bramer WM, Nijs J. Lack of evidence for central sensitization in idiopathic, non-traumatic neck pain: a systematic review. Pain Physician 18:223-36, 2015.

59. Malfliet A, Kregel J, Coppieters I, De Pauw R, Meeus M, Roussel N, Cagnie B, Danneels L, Nijs J. Effect of pain neuroscience education combined with cognition-targeted motor control training on chronic spinal pain: a randomized clinical trial. JAMA Neurol 75:808-17, 2018. https://doi.org/10.1001/jamaneurol.2018.0492 
60. Mason KJ, O'Neill TW, Lunt M, Jones AKP, McBeth J. Psychosocial factors partially mediate the relationship between mechanical hyperalgesia and self-reported pain. Scand J Pain 18:59-69, 2018. https://doi.org/10.1515/sjpain-2017-0109

61. Meeus M, Nijs J. Central sensitization: a biopsychosocial explanation for chronic widespread pain in patients with fibromyalgia and chronic fatigue syndrome. Clin Rheumatol 26:465-73, 2007. https://doi.org/10.1007/s10067-006-0433-9

62. Moher D, Liberati A, Tetzlaff J, Altman DG. Preferred reporting items for systematic reviews and meta-analyses: the PRISMA statement. PLoS Med 6:e1000097, 2009. https://doi.org/10.1371/journal.pmed.1000097

63. Muñoz-García D, López-de-Uralde-Villanueva I, Beltrán-Alacreu H, La Touche R, Fernández-Carnero J. Patients with concomitant chronic neck pain and myofascial pain in masticatory muscles have more widespread pain and distal hyperalgesia than patients with only chronic neck pain. Pain Med 18:526-37, 2017. https://doi.org/10.1093/pm/pnw274

64. Neziri AY, Curatolo M, Nuesch E, Scaramozzino P, Andersen OK, Arendt-Nielsen L, Juni P. Factor analysis of responses to thermal, electrical, and mechanical painful stimuli supports the importance of multi-modal pain assessment. Pain 152:1146-55, 2011. https://doi.org/10.1016/j.pain.2011.01.047

65. Nielsen PK, Andersen LL, Olsen HB, Rosendal L, Sjøgaard G, Sjøgaard K. Effect of physical training on pain sensitivity and trapezius muscle morphology. Muscle Nerve 41:83644, 2010. https://doi.org/10.1002/mus.21577

66. Nijs J, Van Houdenhove B, Oostendorp RA. Recognition of central sensitization in patients with musculoskeletal pain: application of pain neurophysiology in manual therapy practice. Man Ther 15:135-41, 2010. https://doi.org/10.1016/j.math.2009.12.001 
67. Nijs J, Malfliet A, Ickmans K, Baert I, Meeus M. Treatment of central sensitization in patients with 'unexplained' chronic pain: an update. Expert Opin Pharmacother 15:1671-83, 2014. https://doi.org/10.1517/14656566.2014.925446

68. Nijs J, Loggia ML, Polli A, Moens M, Huysmans E, Goudman L, Meeus M, Vanderweeen L, Ickmans K, Clauw D. Sleep disturbances and severe stress as glial activators: key targets for treating central sensitization in chronic pain patients? Expert Opin Ther Targets 21:817-26, 2017. https://doi.org/10.1080/14728222.2017.1353603

69. Nijs J, Elma O, Yilmaz ST, Mullie P, Vanderween L, Clarys P, Deliens T, Coppieters I, Weltens N, Van Oudenhove L, Malfliet A. Nutritional neurobiology and central nervous system sensitisation: missing link in a comprehensive treatment for chronic pain? $\mathrm{Br} \mathrm{J}$ Anaesth, 2019. https://doi.org/10.1016/j.bja.2019.07.016

70. Nir RR, Yarnitsky D. Conditioned pain modulation. Curr Opin Support Palliat Care 9:1317, 2015. https://doi.org/10.1097/spc.0000000000000126

71. Petrini L, Matthiesen ST, Arendt-Nielsen L. The effect of age and gender on pressure pain thresholds and suprathreshold stimuli. Perception 44:587-96, 2015. https://doi.org/10.1068/p7847

72. Piña-Pozo F, Heredia-Rizo AM, Madeleine P, Escobio-Prieto I, Luque-Carrasco A, OlivaPascual-Vaca A. Local and widespread pressure pain hyperalgesia is not side specific in females with unilateral neck pain that can be reproduced during passive neck rotation. J Clin Med 8, 2019. https://doi.org/10.3390/jcm8081246

73. Reeves B.C. DJJ, Higgins J.P.T., Wells G.A. on behalf of the Cochrane Non-Randomised Studies Methods Group. Chapter 13: including non-randomized studies. In: Higgins J.P.T. WGA, editor. Cochrane handbook for systematic reviews of interventions version 510 [updated March 2011]: The Cochrane Collaboration; 2011. Available from: www.cochranehandbook.org. 
74. Rehfuess EA, Akl EA. Current experience with applying the GRADE approach to public health interventions: an empirical study. BMC Public Health 13:9, 2013. https://doi.org/10.1186/1471-2458-13-9

75. Roh YH, Koh YD, Kim JO, Lee KH, Gong HS, Baek GH. Preoperative pain sensitization is associated with postoperative pillar pain after open carpal tunnel release. Clin Orthop Relat Res 476:734-40, 2018. https://doi.org/10.1007/s11999.0000000000000096

76. Rosendal L, Larsson B, Kristiansen J, Peolsson M, Søgaard K, Kjaer M, Sørensen J, Gerdle B. Increase in muscle nociceptive substances and anaerobic metabolism in patients with trapezius myalgia: microdialysis in rest and during exercise. Pain 112:324-34, 2004. https://doi.org/10.1016/j.pain.2004.09.017

77. Sá S, Silva AG. Repositioning error, pressure pain threshold, catastrophizing and anxiety in adolescents with chronic idiopathic neck pain. Musculoskelet Sci Pract 30:18-24, 2017. https://doi.org/10.1016/j.msksp.2017.04.011

78. Scott D, Jull G, Sterling M. Widespread sensory hypersensitivity is a feature of chronic whiplash-associated disorder but not chronic idiopathic neck pain. Clin J Pain 21:175-81, 2005. 79. Sevel L, Boissoneault J, Alappattu M, Bishop M, Robinson M. Training endogenous pain modulation: a preliminary investigation of neural adaptation following repeated exposure to clinically-relevant pain. Brain Imaging Behav, 2019. https://doi.org/10.1007/s11682-018$\underline{0033-8}$

80. Shahidi B, Curran-Everett D, Maluf KS. Psychosocial, physical, and neurophysiological risk factors for chronic neck pain: a prospective inception cohort study. J Pain 16:1288-99, 2015. https://doi.org/10.1016/j.jpain.2015.09.002

81. Shahidi B, Maluf KS. Adaptations in evoked pain sensitivity and conditioned pain modulation after development of chronic neck pain. Biomed Res Int 2017:8985398, 2017. https://doi.org/10.1155/2017/8985398 
82. Sjörs A, Larsson B, Persson AL, Gerdle B. An increased response to experimental muscle pain is related to psychological status in women with chronic non-traumatic neck-shoulder pain. BMC Musculoskelet Disord 12:230, 2011. https://doi.org/10.1186/1471-2474-12-230

83. Smart KM, Blake C, Staines A, Doody C. Self-reported pain severity, quality of life, disability, anxiety and depression in patients classified with 'nociceptive', 'peripheral neuropathic' and 'central sensitisation' pain. The discriminant validity of mechanisms-based classifications of low back (+/-leg) pain. Man Ther 17:119-25, 2012. https://doi.org/10.1016/j.math.2011.10.002

84. Steinmetz A, Jull GA. Sensory and sensorimotor features in violinists and violists with neck pain. Arch Phys Med Rehabil 94:2523-8, 2013. https://doi.org/10.1016/j.apmr.2013.04.019

85. Sterling M. Testing for sensory hypersensitivity or central hyperexcitability associated with cervical spine pain. J Manipulative Physiol Ther 31:534-9, 2008. https://doi.org/10.1016/j.jmpt.2008.08.002

86. Suokas AK, Walsh DA, McWilliams DF, Condon L, Moreton B, Wylde V, Arendt-Nielsen L, Zhang W. Quantitative sensory testing in painful osteoarthritis: a systematic review and $\begin{array}{llll}\text { meta-analysis. } & \text { Osteoarthritis } & \text { Cartilage } & 2012 \text { : }\end{array}$ https://doi.org/10.1016/j.joca.2012.06.009

87. Tampin B, Slater H, Hall T, Lee G, Briffa NK. Quantitative sensory testing somatosensory profiles in patients with cervical radiculopathy are distinct from those in patients with

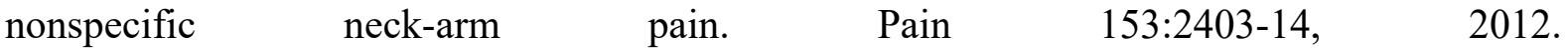
https://doi.org/10.1016/j.pain.2012.08.007

88. Terry EL, France CR, Bartley EJ, DelVentura JL, Kerr KL, Vincent AL, Rhudy JL. Standardizing procedures to study sensitization of human spinal nociceptive processes: Comparing parameters for temporal summation of the nociceptive flexion reflex (TS-NFR). 
Int

https://doi.org/https://doi.org/10.1016/j.ijpsycho.2011.06.021

89. Uddin Z, MacDermid JC. Quantitative sensory testing in chronic musculoskeletal pain. Pain Med 17:1694-703, 2016. https://doi.org/10.1093/pm/pnv105

90. Uthaikhup S, Prasert R, Paungmali A, Boontha K. Altered pain sensitivity in elderly women with chronic neck pain. PLoS One 10:e0128946, 2015. https://doi.org/10.1371/journal.pone.0128946

91. Van Oosterwijck J, Nijs J, Meeus M, Paul L. Evidence for central sensitization in chronic whiplash: a systematic literature review. Eur J Pain 17:299-312, 2013. https://doi.org/10.1002/j.1532-2149.2012.00193.x

92. Wan X, Wang W, Liu J, Tong T. Estimating the sample mean and standard deviation from the sample size, median, range and/or interquartile range. BMC Med Res Methodol 14:135, 2014. https://doi.org/10.1186/1471-2288-14-135

93. Weir CJ, Butcher I, Assi V, Lewis SC, Murray GD, Langhorne P, Brady MC. Dealing with missing standard deviation and mean values in meta-analysis of continuous outcomes: a systematic review. BMC Med Res Methodol 18:25, 2018. https://doi.org/10.1186/s12874-018$\underline{0483-0}$

94. Wells G SB, O'Connell D, Peterson J, Welch V, Losos M, Tugwell P. The NewcastleOttawa Scale (NOS) for assessing the quality if nonrandomized studies in meta-analyses 2012 [Available from: http://www.ohri.ca/programs/clinical_epidemiology/oxford.asp.

95. Woolf CJ, Wall PD. Relative effectiveness of C primary afferent fibers of different origins in evoking a prolonged facilitation of the flexor reflex in the rat. J Neurosci 6:1433-42, 1986. 96. Woolf CJ. Central sensitization: implications for the diagnosis and treatment of pain. Pain 152:S2-15, 2011. https://doi.org/10.1016/j.pain.2010.09.030 
97. Yarnitsky D, Bouhassira D, Drewes AM, Fillingim RB, Granot M, Hansson P, Landau R, Marchand S, Matre D, Nilsen KB, Stubhaug A, Treede RD, Wilder-Smith OH. Recommendations on practice of conditioned pain modulation (CPM) testing. Eur J Pain 19:805-6, 2015. https://doi.org/10.1002/ejp.605

98. Zhu GC, Bottger K, Slater H, Cook C, Farrell SF, Hailey L, Tampin B, Schmid AB. Concurrent validity of a low-cost and time-efficient clinical sensory test battery to evaluate somatosensory dysfunction. Eur J Pain 23:1826-38, 2019. https://doi.org/10.1002/ejp.1456 


\section{Figure legends}

Figure 1. A flowchart showing the process of selection of studies for the review

Figure 2. Meta-analysis of lower pressure pain threshold (mechanical hyperalgesia) at the remote non-painful site in patients with non-traumatic neck pain compared to healthy controls (A), and in patients with moderate/severe non-traumatic neck pain and disability (B) compared to healthy controls (testing locations of both studies in figure 3B were tibialis anterior).

The green square with a line represents the effect size and $95 \%$ confidence interval of each study, with a larger size of the square representing more weight given to the study because of a larger sample size. The position of the diamond represents the pooled effect size while the width of the diamond represents the $95 \%$ confidence interval.

Figure 3. Meta-analysis of higher cold pain threshold (cold hyperalgesia) (A), lower heat pain threshold (heat hyperalgesia) (B) at the remote non-painful site, and lower efficacy of conditioned pain modulation (absolute changes of pressure pain thresholds) (C) in patients with non-traumatic neck pain compared to healthy controls.

The green square with a line represents the effect size and $95 \%$ confidence interval of each study, with a larger size of the square representing more weight given to the study because of a larger sample size. The position of the diamond represents the pooled effect size while the width of the diamond represents the $95 \%$ confidence interval.

Figure 4. Publication bias funnel plots for pressure (A), cold (B) and heat pain thresholds (C) at the non-painful remote site, and conditioned pain modulation (D). $\mathrm{SMD}=$ Standardized mean difference, $\mathrm{SE}=$ Standard error. 


\section{Table Legends}

Table 1. An example of the search strategy used in Pubmed to identify potential articles for screening. Specific search terms, and combinations of terms are presented. There were no limits of language and publication dates. Similar search strategies were conducted in the other databases.

Table 2. Characteristics of included studies with data expressed as mean $\pm \mathrm{SD}$, median (interquartile range) or median [minimum-maximum].

Table 3. Quality appraisal of the reviewed studies.

Table 4. Summary of evidence for altered central pain processing in individuals with nontraumatic neck pain compared to healthy individuals. Studies commenced with low quality and were downgraded or upgraded based on criteria. 